Topics

\title{
Marco Polo - A Mission to Return a Sample from a Near-Earth Object - Science Requirements and Operational Scenarios
}

\author{
By Detlef KoschnY ${ }^{1)}$, Antonella Barucci ${ }^{2)}$, Makoto YoshiKawa ${ }^{3)}$, Hermann BöHnhardT ${ }^{4)}$, John BruCATO ${ }^{5)}$, Marcello \\ CoradinI $^{6}$, Elisabetta DotTo ${ }^{7)}$, Ian Franchi ${ }^{8)}$, Simon F. Green ${ }^{8)}$, Jean-Luc JosseT ${ }^{9}$, Junichiro KaWAGUChI ${ }^{3)}$, Patrick Michel ${ }^{10)}$, \\ Karri MuINONEN $^{11)}$, Jürgen OBERST ${ }^{12)}$, Hajime YANO ${ }^{3)}$, Richard BINZEL ${ }^{13)}$, David AGNOLON ${ }^{1)}$ and Jens RomSTEDT ${ }^{1)}$ \\ ${ }^{1)}$ European Space Agency/ESTEC, Noordwijk, The Netherlands \\ ${ }^{2)}$ LESIA, Paris Observatory, Paris, France \\ 3) JSPEC/ JAXA, Tokyo, Japan \\ ${ }^{4)}$ Max-Planck-Institute for Solar System Research, Katlenburg-Lindau, Germany \\ ${ }^{5)}$ INAF-OAA, Florence, Italy \\ ${ }^{6}$ European Space Agency/HQ, Paris, France \\ ${ }^{7)}$ INAF-OAR, Rome, Italy \\ ${ }^{8)}$ The Open University, Milton Keynes, United Kingdom \\ ${ }^{9)}$ Space Exploration Institute, Neuchâtel, Switzerland \\ ${ }^{10)}$ Univ. Nice Sophia-Antipolis, Obs. de la Côte d'Azur, Nice, France \\ ${ }^{11)}$ Univ. Helsinki Observatory, Helsinki, Finland \\ ${ }^{12)}$ Institute for Planetary Sciences, DLR Berlin, Germany \\ ${ }^{13)}$ Massachusetts Institute of Technology, Cambridge, U.S.A.
}

(Received August 18th, 2009)

\begin{abstract}
Marco Polo is a mission to return a sample from a near-Earth object of primitive type (class C or D). It is foreseen as a collaborative effort between the Japan Aerospace Exploration Agency (JAXA) and the European Space Agency (ESA). Marco Polo is currently in a Phase-A study (status as of summer 2009). This paper focuses on the scientific requirements provided to the industrial study consortia in Europe as well as the possible mission scenario at the target object in order to achieve the overall mission science objectives. The main scientific reasons for going to a near-Earth object are to understand the initial conditions and evolution history of the solar nebula, to understand how major events (e.g. agglomeration, heating) influence the history of planetesimals, whether primitive class objects contain presolar material, what the organics were in primitive materials, how organics could shed light on the origin of molecules necessary for life, and what the role of impacts by NEOs would be in the origin and evolution of life on Earth.
\end{abstract}

Key Words: Marco Polo, Asteroid Sample Return, Science Goals, Mission Design

\section{Introduction}

In response to a call for mission proposals as part of the European Space Agency's (ESA) Cosmic Vision Programme, soliciting ideas for scientific space missions with a possible launch date in the time frame 2015-2025, a team of more than 400 scientists led by A. Barucci and M. Yoshikawa proposed a mission to return a sample from a near-Earth object of primitive type (spectral class $\mathrm{C}$ or $\mathrm{D}$ ) to the Earth ${ }^{1)}$. This would allow the detailed analysis of this sample in ground-based laboratories, which are more capable than any space instrument. In the baseline proposal, this would be a collaboration between the Japanese Aerospace Exploration Agency JAXA and ESA.

The following paper summarizes the science and mission objectives of Marco Polo. It focuses on the scientific requirements provided to the European industrial study consortia, and the mission scenarios at the target object, which are required to achieve the overall mission science requirements. The results of an internal ESA study, which was performed in spring 2008, and the advantages of a collaborative mission between JAXA and ESA are described.

\section{Science Objectives}

Near-Earth objects (NEOs) are part of the small body population in the solar system, which are leftover building blocks of the solar system formation process. They offer important clues to the chemical mixture from which planets formed about 4.6 billion years ago. Studying samples from a NEO will be an excellent opportunity to study the formation and the evolution of the solar system, and the potential contribution of NEOs to the formation of life.

The main objective of the mission is:

To return a sample from a near-Earth object belonging to a primitive class to the Earth.

This will allow the study of the formation of the solar system and the planets, characterisation of a near-Earth object as a representative of a primitive solar system body, and contribute to the field of astrobiology. In addition, scientific information shall be collected to provide the context of the sample.

The detailed scientific objectives are: 
(a) What were the initial conditions and evolution history of the solar nebula?

(b) Which were the properties of the building blocks of the terrestrial planets?

(c) How did major events (e.g. agglomeration, heating) influence the history of planetesimals?

(d) Do primitive class objects contain presolar material yet unknown in meteoritic samples?

(e) What are the organics in primitive materials?

(f) How can organics in NEOs shed light on the origin of molecules necessary for life?

(g) What is the role of impacts by NEOs in the origin and evolution of life on Earth?

All of these objectives are explained in more detail in the proposal which was sent to the European Space Agency by a common European and Japanese proposal team ${ }^{1)}$.

\section{Mission Objectives}

In addition to the science objectives as given above, the technical studies were driven by the following prioritized mission objectives:

i. To enable the safe operation and maneuvering of the spacecraft in close proximity to the NEO and safe collection of the sample.

ii. To place the sample in its global and local context.

iii. To provide complementary science results not achievable from the sample itself.

\section{Detailed Science Requirements}

The so-called Science Requirements Document ${ }^{2)}$ derives science requirements as studied by the ESA Science Study Team from the scientific objectives. These requirements are the formal top-level requirements for the study contractors in European industry and the science community and define in detail what the scientific accomplishments of the space mission shall be. The following sub-sections will give a top-level summary of these science requirements. Note that at the current phase of the study, the science requirements for the ground-based analyses of the sample are not yet given in detail, the focus lies on the science requirements of the space segment.

We first summarize requirements for target selection and the size and properties of the returned sample. After that, the required characterization of the target needed to place the sample in its global and local context is described. There, three phases are distinguished:

- Global characterization' means to measure the properties of the whole NEO, on a global scale;

- 'Local characterization' is the characterization of up to 5 dedicated areas which are identified as potential sampling sites;

- 'Sample context' are measurements being performed at the actual sampling site.

Table 1 gives an overview of the required orders of measurement resolution for the different scenarios.
Table 1. Resolution requirements for global characterization, local characterization, and context measurements.

\begin{tabular}{|l|l|l|l|}
\hline & $\begin{array}{l}\text { Spatial } \\
\text { resolution } \\
\text { for imaging } \\
\text { in the visual }\end{array}$ & $\begin{array}{l}\text { Spatial } \\
\text { resolution for } \\
\text { VIS/IR } \\
\text { spectrometer }\end{array}$ & $\begin{array}{l}\text { Spatial } \\
\text { resolution } \\
\text { for mid-IR } \\
\text { instrument }\end{array}$ \\
\hline $\begin{array}{l}\text { Global } \\
\text { characterisation }\end{array}$ & Order of $\mathrm{dm}$ & Order of $\mathrm{m}$ & Order of $10 \mathrm{~m}$ \\
\hline $\begin{array}{l}\text { Local } \\
\text { characterisation }\end{array}$ & Order of $\mathrm{mm}$ & Order of $\mathrm{dm}$ & Order of $\mathrm{dm}$ \\
\hline $\begin{array}{l}\text { Context } \\
\text { measurements }\end{array}$ & $100 \mu \mathrm{m}$ & - & - \\
\hline
\end{tabular}

\subsection{Requirements related to target selection}

The main scientific goal is to return a sample from a NEO of primitive type. This constrains the taxonomic class of the target. We thus require the target to be a NEO of type C or D according to the Tholen taxonomy ${ }^{3)}$. Subtypes of these as well as B or T are acceptable, as all of these types are expected to consist of primitive material.

There is no strong scientific requirement for a minimum target size. However, the target should be of a size such that:

i) it has sufficient gravity to allow the determination of the gravity field to an accuracy good enough to provide some constraint to the internal structure (e.g. determine the $\mathrm{J} 2$ coefficient to $10 \%$ ).

ii) it is bright enough for fundamental properties (size, shape, albedo, rotation) to be estimated from ground-based observations.

As no precise numbers can currently be given for the above points, for the purpose of this study a minimum absolute visual magnitude of $H \leq 21 \mathrm{mag}$ shall be assumed, corresponding to a diameter $\mathrm{D} \geq 340 \mathrm{~m}$ for a representative primitive body assuming a visual geometric albedo of 0.06 .

To properly perform mission planning, it is important that asteroid size and shape are known at least roughly. Also, its rotation period must be known. If it rotates too fast, it will be difficult to approach one particular point on the NEO and complete the sampling activity without coming onto the night side of the object. Additionally, very fast rotators may have ejected away all their loose regolith and consist of competent rock surface only. Thus, fast rotators should be avoided and we require a minimum rotation period of $\sim 2.5$ hours.

On the upper end of the rotation period, there again is no strong scientific requirement. However, as was shown in the internal ESA study, the longer the rotation period, the longer it will take to map the complete asteroid from orbit. We thus require that the rotation period should not exceed 5 days.

\subsection{Sample requirements}

\subsubsection{Sample mass}

Certain analysis techniques envisaged for the analysis of NEO material, like transmission and reflection spectroscopy or fluorescence analyses can already be done with a few milligrams of sample mass. However, for dating or rare isotope analyses, tens to hundreds of milligrams of a specific 
phase or component are required for each analysis. As multiple analyses are required, and each sample will most likely be a mixture of useful and less useful components and minerals, hundreds of milligrams of each sample are required for this type of work. Detailed understanding of the mineral chemistry, mineral relationships and the isotopic composition of specific phases usually requires preparation of very flat, polished, slices of the rock samples - where gram amounts are required to give a good understanding of the relationships between and within components. Investigation of the organic materials present in the samples will require a battery of instrumentation with a wide range of sample requirements up to grams of bulk sample for isotopic measurements of individual, astrobiologically important molecules such as amino acids and nucleobases as well as structural determination of the large macromolecule.

In addition, many laboratories will be involved in the sample analysis, each laboratory focusing on their special field of interest. Samples have to be selected, sub-divided for integrated studies, prepared (e.g. slices, powders, chips, etc) and distributed.

It will also be necessary to preserve a portion of the sample for future study - in order to exploit new generations of instrumentation, and potentially to address new science questions.

The final science requirement is to return a sample mass of several tens of grams.

\subsubsection{Particle size distribution}

The NEO surface is expected to be covered with regolith, i.e. a mix of loose particles of different sizes. While the smallest particles are likely to be most abundant and represent a good mix of the expected surface material, they will also be the most radiation-damaged. In particular if these particles are sub-millimeter in size, this may become an issue. Larger particles, here called 'fragments' of several $\mathrm{mm}$ in size, however, can be cut apart and in the interior will be free of radiation-induced changes. Thus, one of the science requirements reads "The sampling device shall have the capability to acquire a selection of $\mathrm{cm}$-sized fragments, plus a large amount (ca. 10 $\left.0^{4}\right)$ of small (100s of $\mu \mathrm{m}$-sized to mms-sized) particles." Note that this is a requirement on the design of the sampling mechanism - obviously, if no fragments are on the target's surface, it would be acceptable to return only dust, or vice-versa.

\subsubsection{Additional requirements}

Additional requirements are:

- It shall be possible to perform multiple (up to 3) sampling attempts.

- A verification method shall allow checking whether a sample was taken.

- After collection, the maximum temperature reached by the sample should not exceed $+40{ }^{\circ} \mathrm{C}$ for long durations. For short durations of less than 1 minute, a temperature of up to $+80{ }^{\circ} \mathrm{C}$ is acceptable. This is linked to the fact that for an asteroid coming as close to the Sun as Venus, according to modeling results the temperature about $10 \mathrm{~cm}$ depth will never be above $40^{\circ} \mathrm{C}$. Of course, mixing may have occurred and even material sampled from such a depth may have seen higher temperatures in its history. Still, it should be avoided to modify the sample after sampling.

- Contamination has to be controlled. While Planetary Protection is not an issue for the NEOs considered here (the case was presented to the COSPAR (Committee on Space Research) Planetary Protection panel in 2008 and they have classified Marco Polo as 'unrestricted return'), for scientific reasons any contamination of the sample material with material from the Earth must be avoided, both when manufacturing the spacecraft and after sample return when handling the samples. When descending to the NEOs surface, contamination of the sample by the propulsion system must be minimized. Koschny et al. ${ }^{2)}$ give maximum limits for dust, liquid, and organic contamination of the sample and require tracking possible contaminants with witness plates.

\subsubsection{Putting the samples into context}

While it is expected that most NEOs have a fairly homogeneous surface, it is still important to make a conscious decision on the sampling location(s). The following sections describe in more detail the requirements on the characterization of the complete object which is mainly focused on making this decision possible. From a mission design point of view, it shall be possible to characterize up to five potential sampling sites before the actual sampling. "Characterize" means:

(a) Determine the particle size distribution of the regolith down to scales in the order of millimeters;

(b) Determine the rough mineralogical composition in the order of a decimeter;

(c) Determine the thermal skin depth indicative of regolith properties.

The complete characterization is a three-tier process: at first a global characterization of the complete object with low and medium resolution, then a high-resolution local characterization of up to five potential sampling sites. This will allow the selection of the sampling site (including back-up alternatives). At the actual sampling location, very high resolution context measurements shall be performed.

\subsection{Global characterisation requirements}

'Global characterisation' means to measure the properties pertaining to the entire NEO. This is important to put the sample into context - a crater count on the surface for example would allow an estimate of the age of the object ${ }^{4}$; using infrared spectroscopy one can determine the global distribution of minerals on the surface and compare this with the composition of the sample, and more.

\subsubsection{Imaging requirements}

For Marco Polo, it is required that the complete (visible and illuminated) surface of the NEO will be imaged in at least three different colours, in the visible range with a spatial resolution of the order of decimetres, and with local solar elevation angle between 30 and $60^{\circ}$. This solar elevation will avoid extensive shadowing of some of the surface regions, but give enough shadows to properly determine craters, boulders, 
and other surface features like scarps which will allow to determine the geologic history of the object. It is acknowledged that depending on the rotation axis of the asteroid there may be areas which cannot be imaged due to illumination constraints - it may not be possible to measure in these areas at all.

\subsubsection{Spectroscopy}

The complete surface of the NEO shall be imaged in the visible and near-IR wavelength range from 0.4 to $3.3 \mu \mathrm{m}$ to characterise the mineral properties of the surface with a spectral resolution of $\lambda / \Delta \lambda$ (where $\lambda$ is the wavelength) in the order of 200 and a spatial resolution of the order of meters. If there are areas which are never illuminated it may not be possible to map them. However, in particular the mid-IR observations will also return good results on the dark side of the object.

\subsubsection{Mass, size, and shape}

Part of the global characterisation is the precise determination of the size and shape and rotation period/axis of the object. Once the size and shape is precisely known, measurements of the spacecraft movement will allow the determination of the mass of the object, plus possibly higher-order terms of the gravity field. The knowledge of both mass and shape of the object will provide an estimate of the bulk density of the object. Comparing this value to meteorite analogues will allow constraining the internal structure (porosity) of the object.

To determine the mass of the object to an accuracy of about $1 \%$, the size and shape of the object needs to be known with a certain accuracy. After some analysis, we require for Marco Polo that the shape model must be obtained with an accuracy of typically $1 \mathrm{~m}$ in height and spatial resolution with respect to the centre of mass (in both illuminated and un-illuminated regions). The spatial resolution of the local topography (i.e. in relative coordinates) should be determined to an accuracy of the order of decimetres.

The shape can be determined by a combination of different techniques, namely limb profile measurements, local shape reconstruction from optical stereo observations and/or 'shape from shading', and, the most direct method, by laser altimetry. The laser altimeter will be important to give an absolute number for the distance to the object, which is difficult to obtain from purely optical observations ${ }^{5)}$. However, initial orbit analyses show that it will be difficult to map the complete asteroid surface to the required grid density of $1 \mathrm{~m}$, thus laser measurements will have to be filled in by the other techniques.

\subsubsection{Surface temperature}

To understand the evolution of the sample material over time, it is important to understand the thermal environment of the sample. Both minimum and maximum temperatures that the sample currently sees and the thermal evolution during the 'asteroid year' and over its evolutional history are important.

While the latter can only be assessed through the identification of the source region of the object and by computing its orbital and thermal history from this region to its current orbit ${ }^{6,7)}$, the first two points can be directly measured using infrared observations.
For Marco Polo, we require that the surface temperature can be derived to an accuracy of at least $5 \mathrm{~K}$ (goal $1 \mathrm{~K}$ ) at a spatial resolution of the order of $10 \mathrm{~m}$ at a number of rotational phases from which the thermal inertia can be determined to a precision of better than $10 \%$.

From this, a more detailed measurement requirement is derived - the complete surface of the NEO shall be imaged in the mid-IR with a spatial resolution of the order of $10 \mathrm{~m}$ or better with a spectral resolution of at least $\Delta \lambda / \lambda$ in the order of 200 to determine the wavelength dependent emissivity, and hence identify mineral features in the range $8-16 \mu \mathrm{m}$ (goal 5 $-25 \mu \mathrm{m})$.

\subsubsection{Particle measurements in the NEO environment}

The sample has been exposed to the radiation environment of the NEO as it moves through the solar system. Solar wind and irradiation (mainly in the ultraviolet) will interact with the NEO surface regolith and sputter off particles. There may even be active release processes from volatiles trapped in the NEO surface. To characterise these processes and thus better understand what the radiation history of the returned sample was, the flux, speed, direction and mass of atomic/molecular particles escaping from the surface shall be measured. The energy range from 0.01 to $1 \mathrm{keV}$ shall be covered with an energy resolution of about $25 \%$ and an angular resolution of $5^{\circ} \times 5^{\circ}$; the particles with energies $<0.01 \mathrm{keV}$ shall be measured with $m / \Delta m$ of about 50 where $m$ is the atomic mass.

\subsection{Local characterisation requirements}

'Local characterisation' is the characterisation of up to 5 dedicated areas which are identified as potential sampling sites before the actual sampling. After a successful sampling, another local characterisation measurement cycle shall be performed to record any changes. The requirements for this cycle are structured in a similar way as for the global characterisation, but they only apply to an area of the size of the expected landing accuracy around the potential sampling sites, and with higher resolution. The following detailed requirements apply:

For engineering purposes, the precise topography of the sampling site must be determined; this is foreseen to be done by imaging. Just as on the global scale, imaging is important for determining the geological context of the sample. In particular, the particle size distribution of the regolith can be determined to ensure that a sample with the envisaged mix of larger fragments and fine dust can be obtained. We thus require imaging in the visible in at least three colour filters, with a spatial resolution of the order of millimetres.

The detailed mineral composition of the surface down to cm-resolution $(\lambda / \Delta \lambda$ in the order of 200$)$ will be determined to allow the study of small-scale variations in the mineralogy. This is again an important measurement to put the sample in context - did it come from a very homogeneous surface area, or a mixed one?

Observations in the mid-IR can, as on the global scale, constrain the thermal properties of the surface but this time on a much smaller spatial scale. Local variations in e.g. the thermal inertia may allow assessing the porosity of the regolith and its variation in the vicinity of the sampling site. For these measurements, we require imaging in the mid-IR 
with a spatial resolution of decimetres and a spectral resolution of at least $\lambda / \Delta \lambda$ in the order of 200 or better, in the range $8-16 \mu \mathrm{m}$ (goal $5-25 \mu \mathrm{m}$ ). In addition, these measure-ments will support the identification of mineral features.

Last but not least we also require the measurement of the atomic particle flux, as in the previous section.

\subsection{Sample context requirements}

These requirements concern measurements which will be performed directly before and after sampling, most likely from the spacecraft in contact with the surface. The first important point is to determine the regolith size distribution of the actual sampling site to very small sizes. We require $100 \mu \mathrm{m}$ resolution (goal $15 \mu \mathrm{m}$ ) in an area about 5 times larger than the area sampled by the sampling device. It is assumed that this is done via a close-up imaging camera. This measurement shall be done before and after the sampling and will record e.g. slumping of material into the hole left by the sampler. This will allow estimating material properties of the surface, like friction coefficients.

From a geology point of view, determining the texture and organisation of the regolith surface is important. Are the large fragments covered by fine dust, are they rounded or sharp-edged, can fractures be seen? Is the particle size distribution homogeneous on the smallest scale, is there layering visible? The same imaging system as mentioned above will make these observations.

As a non mission-critical requirement it is specified that the bulk rock-forming elemental ( $\mathrm{Na}, \mathrm{Mg}, \mathrm{Al}, \mathrm{Si}, \mathrm{P}, \mathrm{S}, \mathrm{Cl}, \mathrm{K}, \mathrm{Mn}$, $\mathrm{Fe}$, and $\mathrm{Ni}$ ) composition of the sampling area should be determined. This could be done with an optional APXS instrument (see Table 2).

\subsection{Other requirements}

The Science Requirements Document ${ }^{2}$ lists a few other requirements which do not fit into the sections as given above. These are summarized here in bullet form.

(a) In-flight calibration of the instruments is an important part of achieving the scientific goals. Thus, calibration targets must be provided. For example, if the mission design would be such that the escape from the Earth is done via a lunar swing-by (which is currently not in the baseline), it should be possible to perform measurements of one of the Apollo landing sites. These are the perfect calibration standards as rocks from that area have been characterised in ground-based labs.

(b) After the sample has been taken, some kind of verification method should be in place to ensure that a sample of sufficient mass was taken.

(c) If the mission scenario foresees any planetary flyby, it shall be possible to switch on all payload for testing.

(d) Determine the J2 terms of the gravitational field. This can be done with precise radio measurements. An important point which still needs to be studied is how long it would take to perform these measurements.

(e) Make images from the star tracker cameras available on ground. These images could be used to remote search for asteroids from a vantage point different from that of the Earth, thus offering views on these objects from phase angles not accessible from the Earth. Typical star trackers are very light-sensitive systems which have a field of view of about $15 \mathrm{deg}$. Using long exposures in the order of $30 \mathrm{~s}$ or longer, objects as faint as $\mathrm{M}=10-12 \mathrm{mag}$ (Bessel V-Band) can be imaged, allowing to monitor the largest asteroids or discover new Inner Earth Objects when the spacecraft is located within the Earth's orbit.

\section{The Internal ESA Assessment Study}

\subsection{Strawman payload}

Most of the scientific requirements can be directly linked to a measurement technique like imaging or spectroscopy. Under the coordination of ESA's Payload Study Manager, a so-called 'strawman payload' was put together which acts as a baseline for the mission development ${ }^{8}$. It does not exclude additional instruments to be included at a later stage of the mission, or even the deletion of instruments if they are deemed unnecessary.

The general rule for the internal ESA assessment study was to keep the payload as focussed as possible on its main objective "To put the returned sample into global context". This is different for the baselined JAXA-ESA mission, where additional instruments can be flown.

Table 2. Strawman payload with optional instruments for the ESA-internal Marco Polo study. Priorities: $1=$ essential, 2 = beneficial, 3 $=$ optional instrument. MEX $=$ Mars Express, VEX $=$ Venus Express, BepiC $=$ BepiColombo.

\begin{tabular}{|c|c|c|c|c|}
\hline Name & Weight & $\begin{array}{l}\text { Power } \\
\text { (avg.) }\end{array}$ & $\begin{array}{l}\text { Flight } \\
\text { heritage }\end{array}$ & Priority \\
\hline $\begin{array}{l}\text { Wide Angle } \\
\text { Camera }\end{array}$ & $1 \mathrm{~kg}$ & $19 \mathrm{~W}$ & $\begin{array}{l}\text { Rosetta, } \\
\text { VEX }\end{array}$ & 1 \\
\hline $\begin{array}{l}\text { Narrow Angle } \\
\text { Camera }\end{array}$ & $5.5 \mathrm{~kg}$ & $22 \mathrm{~W}$ & $\begin{array}{l}\text { Rosetta, } \\
\text { VEX, } \\
\text { BepiC }\end{array}$ & 1 \\
\hline Laser Altimeter & $5.0 \mathrm{~kg}$ & $26 \mathrm{~W}$ & BepiC & 1 \\
\hline $\begin{array}{l}\text { Visible/Near-Infrar } \\
\text { ed Spectrometer }\end{array}$ & $4.0 \mathrm{~kg}$ & $22 \mathrm{~W}$ & $\begin{array}{l}\text { Rosetta, } \\
\text { BepiC } \\
\text { Hayabusa }\end{array}$ & 1 \\
\hline $\begin{array}{l}\text { Mid Infrared } \\
\text { Spectrometer }\end{array}$ & $2.5 \mathrm{~kg}$ & $2 \mathrm{~W}$ & $\mathrm{n} / \mathrm{a}$ & 2 \\
\hline $\begin{array}{l}\text { Radio Science } \\
\text { Experiment }\end{array}$ & $\mathrm{n} / \mathrm{a}$ & $\mathrm{n} / \mathrm{a}$ & $\begin{array}{l}\text { Rosetta, } \\
\text { VEX, MEX }\end{array}$ & 1 \\
\hline $\begin{array}{l}\text { Neutral Particle } \\
\text { Analyser }\end{array}$ & $2.6 \mathrm{~kg}$ & $13 \mathrm{~W}$ & $\begin{array}{l}\text { MEX, } \\
\text { VEX, } \\
\text { BepiC }\end{array}$ & 2 \\
\hline Close-up Camera & $0.5 \mathrm{~kg}$ & $25 \mathrm{~W}$ & ExoMars & 1 \\
\hline $\begin{array}{l}\text { Alpha Particle } \\
\text { X-ray } \\
\text { Spectrometer } \\
\text { (APXS) }\end{array}$ & $0.4 \mathrm{~kg}$ & $1.5 \mathrm{~W}$ & $\begin{array}{l}\text { Mars } \\
\text { Pathfinder, } \\
\text { Mars } \\
\text { Exploration } \\
\text { Rover, } \\
\text { Rosetta }\end{array}$ & 3 \\
\hline $\begin{array}{l}\text { Electric field } \\
\text { sensor }\end{array}$ & $2.0 \mathrm{~kg}$ & $2 \mathrm{~W}$ & ExoMars & 3 \\
\hline $\begin{array}{l}\text { Attenuated Total } \\
\text { Reflection Infrared } \\
\text { Spectrometer }\end{array}$ & $0.2 \mathrm{~kg}$ & $1.2 \mathrm{~W}$ & $\mathrm{n} / \mathrm{a}$ & 3 \\
\hline $\begin{array}{l}\text { Temperature } \\
\text { Sensor }\end{array}$ & $0.3 \mathrm{~kg}$ & $0.5 \mathrm{~W}$ & $\begin{array}{l}\text { Mars } \\
\text { Pathfinder, } \\
\text { ExoMars }\end{array}$ & 3 \\
\hline
\end{tabular}




\subsection{Possible mission scenario}

The ESA-internal mission study focused on a mission with only chemical propulsion; electrical propulsion would offer more flexibility in terms of target selection, however, it was not considered in this study for technical and cost reasons.

Table 3 shows the most feasible targets for a chemical mission and gives a ranking. Four mission scenarios were studied ${ }^{9)}$, two based on a launch into a Geostationary Transfer Orbit (GTO), two based on direct escape from Earth. For both strategies, launches in 2017 to 2020 were analyzed. The main target in these studies was 1989 UQ.

Table 3. Potential mission targets for the ESA-internal mission study.

\begin{tabular}{|l|l|l|}
\hline NEO & $\begin{array}{l}\text { Spectral } \\
\text { type }\end{array}$ & Feasibility \\
\hline 2001 SG286 & D & Difficult \\
\hline 2001 SK162 & T & Medium \\
\hline 1999 JU3 & Cg & Feasible \\
\hline 1989 UQ & Ch & Feasible \\
\hline
\end{tabular}

In the currently ongoing assessment study, $1999 \mathrm{JU} 3$ was selected as the baseline target as it was the most feasible target. The baseline launch date would be in November 2018, with a backup in November 2019.

The launch window would be from 20 Nov 2018 to 10 Dec 2018. There will be two Earth swing-bys, one in Nov/Dec 2019 (depending on the launch date), one in Dec 2020. Instruments can be operated during these swing-bys to perform calibration measurements, e.g. imaging of extended objects, or using the Moon as calibration target.

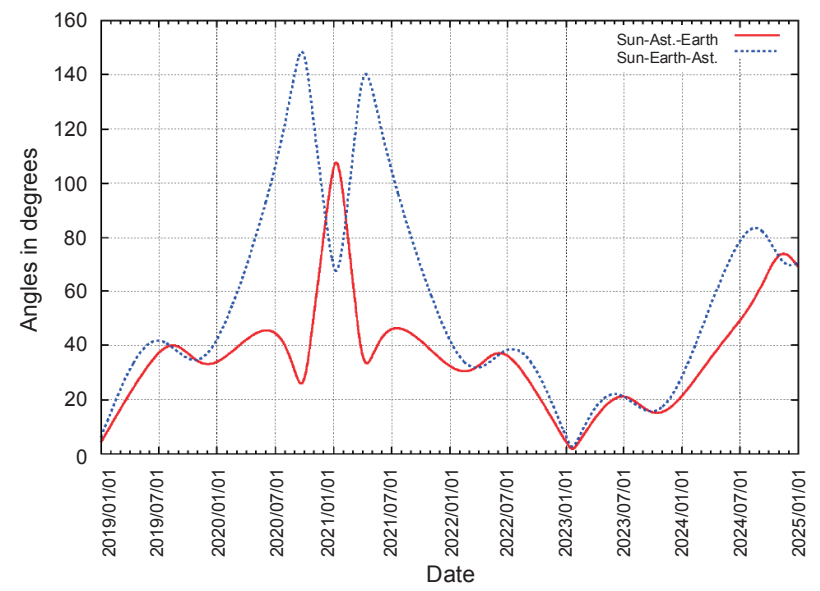

Fig. 1. Solar elongations of 1999 JU3 versus time. At the arrival on 20 Dec 2020, the asteroid is close to the Sun. The $\mathrm{x}$-axis gives the date, starting 2019/01/01, ending at 2025/01/01. The y-axis gives angles in degrees from 0 to $160^{\circ}$.

The spacecraft would arrive at the asteroid in the time frame January to February 2022. There is a superior conjunction in early 2023 which will need to be taken into account in the mission operations planning. During a superior conjunction, the asteroid gets very close to the Sun as seen from the Earth (see Fig. 1), meaning that radio contact is difficult and critical operations have to be avoided.

This mission would be able to bring about $1190 \mathrm{~kg}$ mass to the asteroid. The time between arrival and departure from the asteroid would be 17.5 months. The spacecraft would arrive at the Earth in December 2024 and the Earth Reentry Capsule would enter the Earth's atmosphere at between $11.86 \mathrm{~km} / \mathrm{s}$ and $12.44 \mathrm{~km} / \mathrm{s}$ depending on whether a retrograde or prograde reentry would be chosen.

The backup mission is essentially similar to the baseline, with the first one-year arc removed, which reduces the mission duration to 5 years.

As a backup target, scenarios for 1989 UQ were studied with launches in 2017 and 2018. This target is slightly less favourable as the spacecraft has to perform a Venus swing-by and the thermal design will be more challenging.

Table 4 shows the major milestones of the baseline mission.

Table 4. Mission timeline for the baseline mission.

\begin{tabular}{|l|l|}
\hline $\begin{array}{l}\text { Launch window }- \text { on Soyuz Fregat } \\
\text { launcher }\end{array}$ & $\begin{array}{l}20 \text { November } 2018- \\
10 \text { December } 2018\end{array}$ \\
\hline $\begin{array}{l}\text { Earth swing-by (between } \sim 155700 \mathrm{~km} \text { and } \\
\sim 424000 \mathrm{~km} \text { altitude) }\end{array}$ & $\begin{array}{l}20 \text { November } 2019- \\
10 \text { December } 2019\end{array}$ \\
\hline $\begin{array}{l}\text { Earth swing-by (between } \sim 7100 \mathrm{~km} \text { and } \\
\sim 12330 \mathrm{~km} \text { altitude) }\end{array}$ & 05 December 2020 \\
\hline $\begin{array}{l}\text { Arrival at } 1999 \mathrm{JU} 3 \text {, arrival mass } \sim 1190 \mathrm{~kg} \\
\text { (using conventional propulsion system) }\end{array}$ & $\begin{array}{l}\text { 19 January } 2022- \\
14 \text { February } 2022\end{array}$ \\
\hline Departure from asteroid & 30 July 2023 \\
\hline $\begin{array}{l}\text { Earth atmospheric entry with 11.86 - } \\
12.44 \mathrm{~km} / \mathrm{s}\end{array}$ & 04 Dec 2024 \\
\hline
\end{tabular}

A first top-level design of the spacecraft is shown in Fig. 2. A number of trade-offs were performed to arrive at this configuration. In the first instance, different configurations for propulsion module, main spacecraft, lander, return vehicle, and Earth Return Capsule were studied. This study arrived at a main spacecraft which includes the propulsion system and a separate Earth Return Capsule (ERC) with heat shield. The main spacecraft with the ERC will fly to the asteroid, take the sample and transfer it to the ERC, then return to the Earth and eject the ERC shortly before entering the Earth's atmosphere.

The tank accommodation is driving the overall volume and layout of the spacecraft. The sampling mechanism is at the bottom of the spacecraft to have easy access to the surface. The ERC is mounted on the top to avoid contamination by surface dust, requiring some kind of elevator mechanism to bring the samples into the capsule. No large solar arrays are required as only chemical propulsion is assumed; the solar arrays will be body-mounted. The landing feet are fixed-mounted. In the landed configuration, the spacecraft stands $2.3 \mathrm{~m}$ high. The octagonal structure has a maximum size of $2.8 \mathrm{~m}$.

The assumed mass of the ERC in this study is $75 \mathrm{~kg}$; the assumed payload mass is $\sim 25 \mathrm{~kg}$ including a $20 \%$ margin. The total launch margin is about $25 \%$, including a system margin of $20 \%$. With this, the strawman payload (plus some 
optional instruments) as given in Table 2 can be accommodated. Note that the current studies indicate that an ERC mass of as low as $30 \mathrm{~kg}$ can be achieved if a parachute system is used.
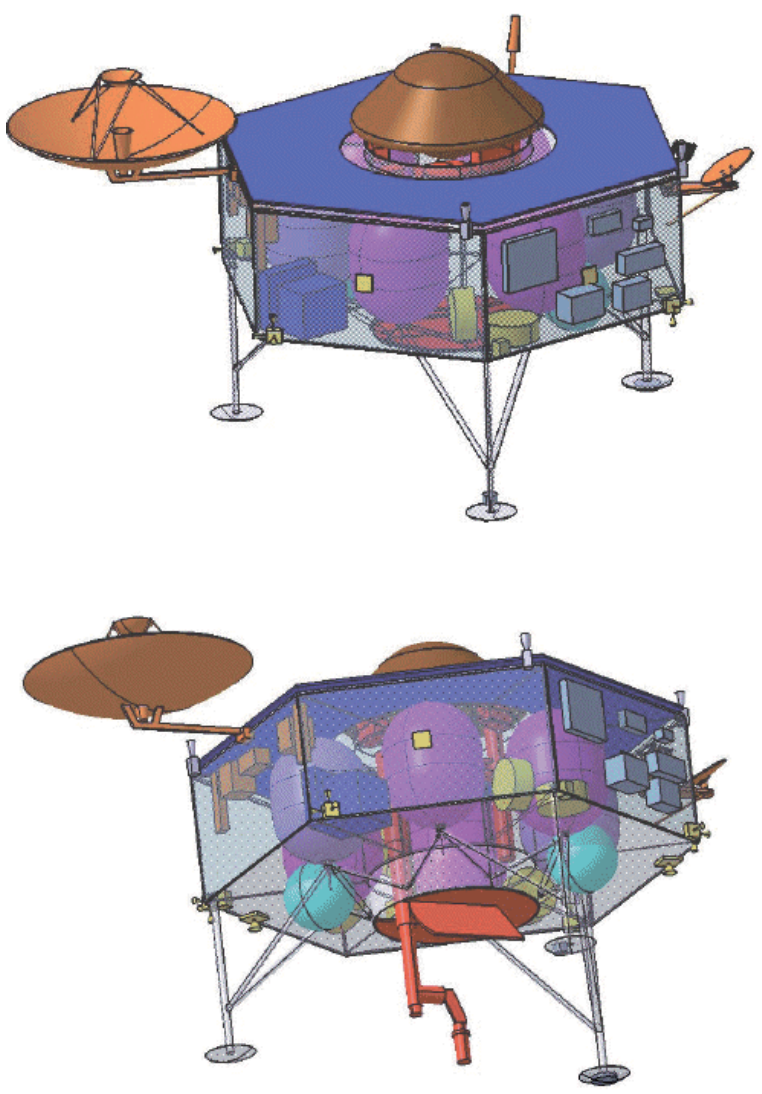

Fig. 2. The Marco Polo spacecraft as designed in the ESA-internal study. The diameter of the hexagon is about $3 \mathrm{~m}$.

\subsection{Operational activities at the NEO}

In the 'Mission 2017', about 18 months are available at the target asteroid. Some of the time will be compromised because of the closeness to solar conjunction. However, almost one and a half years will be available for performing the scientific measurements.

To structure the mission, several mission phases are introduced when in proximity to the NEO:

(a) Formation Flying or Far Global Characterisation (FAR)

(b) Detailed gravity field $=$ Radio Science phase (RSE)

(c) Global characterisation (GLO)

(d) Local characterisation (LOC)

(e) Sampling and sample context measurements (SAM)

(f) Extended global monitoring (EGLO)

FAR: When arriving at the NEO, the spacecraft will - after a first detection of the object with the on-board cameras slowly approach the object. The first observation phase, FAR, will be done from a safe distance to the object (around $5 \mathrm{~km}$ ).

The asteroid will rotate with respect to the spacecraft and most of the asteroid's surface will be visible. The percentage of the visible surface area depends on the position of the rotation axis with respect to the s/c position. Thus, after a first assessment of the rotation axis position, it should be possible to move the s/c to a more optimal position.

The position will be determined by a balance of asteroid gravity, solar radiation pressure, and possibly the perturbing forces from other planets. It will be at the edge or outside the sphere of influence of the asteroid (see Fig. 3).

The observations from this position will provide a first characterisation of the asteroid, both for scientific and for engineering purposes. The main points addressed will be to determine the precise rotational state of the asteroid (period, position of rotation axis, rotation direction, stable/chaotic rotation), to produce a first shape model, and to derive a first mass estimate of the asteroid.

RSE: After a possible waiting phase because of the solar conjunction and an observing phase of about two weeks, the spacecraft will go to an orbit where the gravity field can be mapped using Radio Science. This is an orbit where the perturbance forces on the orbit are minimized, thus allowing best the determination of the gravity field in high detail. This could be a terminator orbit as shown in Figure 3, but possibly also an orbit inclined towards the sun would be feasible.

Note that due to the solar radiation pressure on the spacecraft, it is expected that a possible 'terminator orbit' will not be directly over the terminator, but shifted, in a direction away from the Sun. This means that when pointing to the asteroid, in particular when pointing towards the illuminated side, the Sun will be less than $90^{\circ}$ away from the instrument boresight, i.e. not ideal for remote-sensing observations of the object.

The main goal of this phase is the characterisation of the gravity field, which will be done by precise tracking of the spacecraft from ground. In the ESA-internal study, it is estimated that this phase will take about one month.

GLO: This is the main science orbit where the complete characterisation of the asteroid will be performed. The current assumption is that the spacecraft is in a "9-o'clock orbit", i.e. that the angle between the orbital plane and the plane through the asteroid - Sun line perpendicular to the asteroid orbital plane is $30^{\circ}$. As a result, the sub-spacecraft point will have reasonable illumination conditions for imaging, with the Sun typically between $30^{\circ}$ and $60^{\circ}$ above the horizon, resulting in shadows of surface features which are visible but not too long. Here, most of the science observations to perform the global characterisation will be performed, including mapping in the visible and infrared wavelength bands and mapping the neutral atoms and molecules released from the surface. The laser altimeter will produce a close grid of measurement points with the distance to the asteroid.

It is estimated that about 2 months are needed to get the coverage and resolution requirements as stated before.

LOC: The spacecraft will go close to the asteroid (typically 
$200 \mathrm{~m}$ distance) and use the remote sensing instruments to characterise the potential sampling sites. This will be done for up to 5 potential sampling sites. The spacecraft is assumed to stay still above the potential sampling site for the needed duration of the measurement, currently assumed to be in order of 10 minutes to one hour.

To perform the local characterisation of up to 5 potential sampling sites, a duration of about 5 weeks is estimated.

SAM: During the landing or at least touching the asteroid's surface, the sample context measurements will be performed. The sampling activity itself will only take a few minutes to tens of minutes; however, the complete phase includes the descent and later the ascent and can take several days for one sampling activity.

EGLO: If after the sampling the spacecraft has to wait to be able to return to Earth, the 'extended global monitoring' can be performed. It is assumed that this phase can be done with an orbit similar to the 'global characterisation' phase. However, it is acknowledged that the orbit where this is done from should be a safe orbit from an engineering point of view,

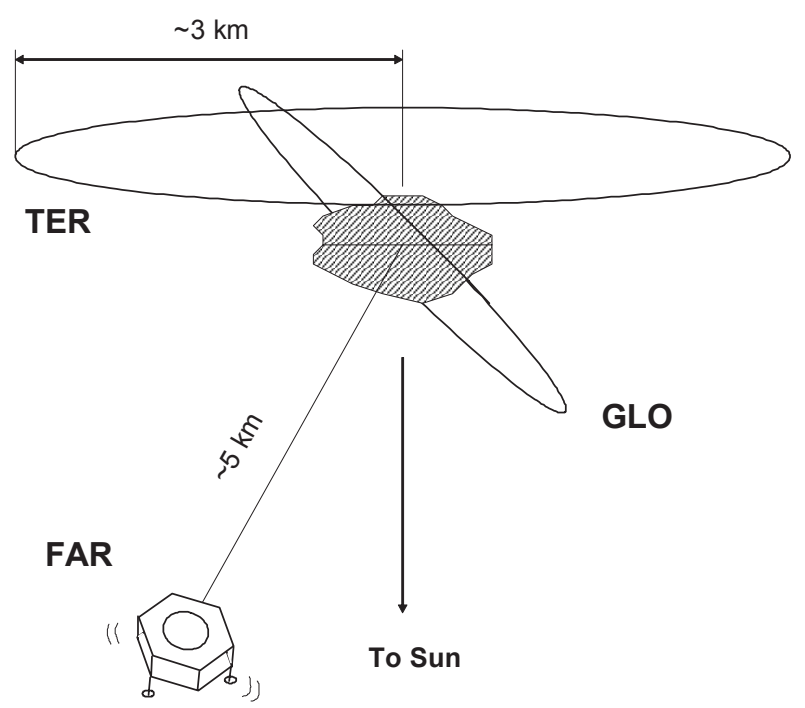

Fig. 3. Possible orbit configuration for the first mission phase. For an explanation of the abbreviations, see the text.

to minimize the risk that the acquired sample cannot be returned to the Earth.

Here science measurements can continue and look at long-term effects on the asteroid, for example

(a) Continue monitoring the asteroid with the IR instruments in regular intervals to study the thermal behaviour of the asteroid at different solar distances;

(b) Monitor the long-term behaviour of particle sputtering from the asteroid surface;

(c) Add data to get a higher-density grid of measurements with the Laser Altimeter;

(d) Add image data to obtain more phase angle coverage and/or stereo information.

\section{A Common JAXA-ESA Mission}

The scenario suggested in the initial proposal is to perform this mission in a collaborative effort between ESA and JAXA. JAXA has gained experience with the asteroid sample-return mission Hayabusa, which rendezvoused with asteroid 25143 Itokawa in mid-September $2005^{10)}$. It is scheduled to deliver its sample to Earth by June 2010;

The proposed task share between JAXA and ESA would be: JAXA will provide the so-called 'mothership', which contains all required propulsion systems and the main sampling system. The mothership will carry a separate Earth Return Capsule provided by ESA and, optionally, a nationally-funded Lander. Europe would also provide support for the mission and science operations and data archiving. The launcher would be provided by ESA.

The payload will be provided by both partners, either via an Announcement of Opportunity process for European and Japanese instruments.

The main difference to the previously described study is that by combining forces, the mission can afford to be done with electrical propulsion and thus be larger, affording to carry a separate lander, and reaching other targets than described above.

\section{Current Status and Future Steps}

The Marco Polo asteroid sample return mission is currently in a Phase-A (assessment) study. In Europe, three industrial consortia study the mission in parallel. In Japan, Marco Polo is being studied in Phase-A. Mutual interface meetings have started to coordinate these studies and converge to a common mission.

In Europe, Marco Polo is studied as part of the Cosmic Vision programme, ESA's long-term programme to define scientific missions for launch in the time frame 2015-2025. Following the schedule of Cosmic Vision, assessment reports for the feasibility and performance of the mission will have to be provided by industry to ESA's advisory structure. Currently, six M class missions are studied as part of Cosmic Vision. The Science Programme Committee of ESA will have to decide in fall 2009 on which missions will continue to be studied in Phase-B. A final selection on the missions to be implemented will take place in fall 2011.

In Jun 2008, ESA issued a call for a 'Declaration of Interest for payload-related studies' where 21 consortia mainly of research institutes sent valid proposals for Marco Polo. There were 18 studies related to scientific instruments (some of them for a Lander), two studies related to curation facilities, and one study for a complete Lander including instrumentation. A total of 12 studies were recommended by an ESA assessment team to be actually performed. The studies themselves are 
funded by the national funding agencies.

These studies are preformed in parallel to the industry studies and provide payload-related input like preliminary instrument design and resource requirements, accommodation requirements, and operational requirements.

\section{Conclusion}

Marco Polo is a study for a common JAXA-ESA mission to return a sample from a primitive near-Earth object. This paper outlines the top-level science goals and breaks them down into mission requirements. The current studies show that the mission is feasible and would be ready for selection for a Phase-B study.

\section{References}

1) Barucci, M.A., Yoshikawa, M., Michel, P., Kawaguchi, J., Yano, H., Brucato, J.R., Franchi, I.A., Dotto, E., Fulchignoni, M. and Ulamec, S.: Marco Polo: near-Earth object sample return mission, Experimental Astronomy, 23, Iss. 3 (2009), pp. 785-808.

2) Koschny, D., Barucci, A., Böhnhardt, H., Brucato, J., Dotto, E., Franchi, I., Green, S., Josset, J.-L, Michel, P., Muinonen, K. and Oberst, J.: Marco Polo Science Requirements Document, MP-RSSD-RS-001/2b, ESA (2009) (available via http://sci.esa.int/marcopolo - navigate to 'documents').

3) Tholen, D. and Barucci, A.: Asteroid taxonomy, in: Asteroids II;
Proceedings of the Conference, Tucson, AZ, Mar. 8-11, 1988 (A90-27001 10-91). Tucson, AZ, University of Arizona Press, 1989, pp. 298-315.

4) Michel, P. and Yoshikawa, M.: Dynamical origin of the Asteroid (25143) Itokawa: the target of the sample return mission Hayabusa, $A \& A, 449$ (2006), pp. 817-820.

5) Gaskell, R., Barnouin-Jha, O., Scheeres, D., Mukai, T., Hirata, N., Abe, S., Saito, J., Hashimoto, T., Ishiguro, M. and Kubota, T. High Resolution Global Topography of Itokawa From Hayabusa Imaging and LIDAR Data, American Geophysical Union, Fall Meeting (2007), abstract \#P32A-01.

6) Bottke, W.J., Morbidelli, A., Jedicke, R., Petit, J.M., Levison, H., Michel, P. and Metcalfe, T.S.: Debiased Orbital and Size Distribution of the near-Earth objects, Icarus, 156 (2002), pp. 399-433.

7) Michel, P., O'Brien, D.P., Abe, S. and Harata, N.: Itokawa's cratering record as observed by Hayabusa: Implications for its age and collisional history, Icarus 200 (2009), pp. 503-513.

8) Romstedt, J. and the Marco Polo Science Study Team: Payload Definition Document (PDD), SCI-PA/2008.002/Marco-Polo, Issue 3.0, 30 Jan 2009 (available via http://sci.esa.int/marcopolo navigate to 'documents').

9) Escorial, D., Walpot, L., Ferracina, L, Drai, R., Burzigotti, P., Barton, A., Pelt, M., Lamboglia, E., Sanchez, A., Timm, R., Coste, P., Palladino, M., Khan, M., De Pascale, P. and Murchdoch, N.: CDF Study Report Marco Polo, CDF-72(A), ESA, May 2008.

10) Yoshikawa, M., Fujiwara, A., Kawaguchi, J.: Hayabusa and its adventure around the tiny asteroid Itokawa, Highlights of Astronomy, 14 (2007), pp. 323-324. 